\title{
Genetics and Biomarkers of Moyamoya Disease: Significance of RNF213 as a Susceptibility Gene
}

\author{
Miki Fujimura, ${ }^{a}$ Shinya Sonobe, ${ }^{\text {a }}$ Yasuo Nishijima, ${ }^{\text {a }}$ Kuniyasu Niizuma, ${ }^{\text {a Hiroyuki Sakata, }}{ }^{a}$ \\ Shigeo Kure, ${ }^{b}$ Teiji Tominaga ${ }^{a}$ \\ Departments of ${ }^{a}$ Neurosurgery and ${ }^{b}$ Pediatrics, Tohoku University Graduate School of Medicine, Sendai, Japan
}

Moyamoya disease is characterized by a progressive stenosis at the terminal portion of the internal carotid artery and an abnormal vascular network at the base of the brain. Although its etiology is still unknown, recent genome-wide and locus-specific association studies identified RNF213 as an important susceptibility gene of moyamoya disease among East Asian population. A polymorphism in c. 14576G > A in RNF213 was identified in 95\% of familial patients with moyamoya disease and 79\% of sporadic cases, and patients having this polymorphism were found to have significantly earlier disease onset and a more severe form of moyamoya disease, such as the presentation of cerebral infarction and posterior cerebral artery stenosis. The exact mechanism by which the RNF213 abnormality relates to moyamoya disease remains unknown, while recent reports using genetically engineered mice lacking RNF213 by homologous recombination provide new insight for the pathogenesis of this rare entity. Regarding biomarkers of moyamoya disease, moyamoya disease is characterized by an increased expression of angiogenic factors and pro-inflammatory molecules such as vascular endothelial growth factors and matrix metalloproteinase- 9 , which may partly explain its clinical manifestations of the pathologic angiogenesis, spontaneous hemorrhage, and higher incidence of cerebral hyperperfusion after revascularization surgery. More recently, blockade of these pro-inflammatory molecules during perioperative period is attempted to reduce the potential risk of surgical complication including cerebral hyperperfusion syndrome. In this review article, we focus on the genetics and biomarkers of moyamoya disease, and sought to discuss their clinical implication.

Keywords Moyamoya disease, Genetics, Biomarkers, RNF213, Susceptibility gene

\author{
Correspondence: Miki Fujimura \\ Department of Neurosurgery, Tohoku \\ University Graduate School of Medicine, \\ 1-1 Seiryo-machi, Aoba-ku, \\ Sendai 980-8574, Japan \\ Tel: +81-22-717-7230 \\ Fax: +81-22-717-7233 \\ E-mail: fujimur@nsg.med.tohoku.ac.jp
}

Received: March 30, 2014 Revised: April 18, 2014

Accepted: April 19, 2014

This study was supported by JSPS KAKENHI Grant Number 24659642.

The authors have no financial conflicts of interest.

\section{Introduction}

Moyamoya disease (MMD) is characterized by a progressive stenosis at the terminal portion of the internal carotid artery (ICA) and an abnormal vascular network at the base of the brain. ${ }^{1,2}$ The etiology of MMD remains unknown, but recent advances have been made in understanding the molecular biology and pathophysiology of this rare entity. Previous studies explored genetic factors and revealed several loci associated with moyamoya disease; 3p24-p26, ${ }^{3} 6 \mathrm{q} 25,{ }^{4} 8 \mathrm{q} 23,{ }^{5}$ and $17 \mathrm{q} 25 .{ }^{6}$ More recently, the RNF213 gene (RNF213) in the 17q25-ter region was identified as a novel susceptibility gene for MMD among East Asian population. ${ }^{7-9}$ A polymorphism in c. $14576 \mathrm{G}>\mathrm{A}$ in RNF213 was identified in $95 \%$ of familial patients with MMD and $79 \%$ of sporadic cases, ${ }^{7}$ and RNF213 was found to correlate with the early-onset and severe forms of MMD, indicating its value as a good biomarker for predicting prognosis. ${ }^{10}$ The exact mechanism by which the RNF213 abnormality relates to moy- 
amoya disease remains unknown, but recent reports using genetically engineered mice lacking RNF213 by homologous recombination provide new prospects for the basic research of this rare entity. ${ }^{11,12}$ In this review article, we focus on the genetics and biomarkers of MMD, and sought to discuss their clinical implication.

\section{Basic pathology of MMD}

\section{Histo-pathological characteristics}

Intimal hyperplasia and medial thinness are well-known histo-pathological characteristics of MMD., 2,13,14 The thickened intima has increased number of smooth muscle cells, which are thought to be synthetic-type smooth muscle cell migrating from medial layer. ${ }^{15}$ Degradation of the smooth muscle cells in the medial layer and subsequent death of the vascular smooth muscle cells are thought to result in the characteristic finding of medial thinness not only at the terminal ICA but also at the peripheral middle cerebral artery. ${ }^{14}$ Together with the waviness and duplication of the internal elastic lamina, intracranial arteries of moyamoya disease generally have intrinsic fragility, which should also be mentioned as one of the pitfalls of revascularization surgery while manipulating peripheral middle cerebral artery during extracranial-intracranial (EC-IC) bypass. ${ }^{16}$

\section{Macroscopic pathology of MMD; physiological reorganization system}

For the better understanding of MMD as dynamic disease, it is particularly important to revisit Suzuki's angiographic staging, ${ }^{1,2}$ which represent the natural course of the angio-architecture in moyamoya patients by physiological reorganization system. Suzuki's angiographic staging does not reflect the severity of MMD, while it explains how 'moyamoya disease' compensates its ischemic condition by physiological process. ${ }^{16}$ At the early stage of MMD, progressive stenosis of the terminal ICA occurs while it is subsequently compensated by abnormal vascular networks at the base of the brain at stage III. In substantial number of patients, ischemic brain is further compensated by trans-dural anastomosis from external carotid system without surgical intervention at stage IV to VI, resulting in the disappearance of moyamoya vessels. We named this physiological reorganization process as internal carotid (IC)-external carotid (EC) conversion., ${ }^{16}$ Concept of revascularization surgery for $M M D$, either direct or indirect revascularization, is to accomplish successful 'IC-EC conversion' to prevent insufficient reorganization resulting in cerebral infarction. Therefore, the concept of revascularization surgery for MMD should be based on the idea to support the intrinsic compensatory nature of MMD, rather than to eradicate the pathophysiology of this entity.

\section{Genetics of MMD}

\section{Background and previous reports}

Since some patients with MMD show autosomal dominant inheritance pattern, genetic factors have been implicated in the etiology of MMD. In fact, gene loci have been identified in 3q24p $26^{3}$ and $8 \mathrm{q} 23^{5}$ in genome-wide analysis, and in $6 \mathrm{q} 25^{4}$ and $17 \mathrm{q} 25^{6}$ in a chromosomal search for familial MMD. The RNF213 in the $17 \mathrm{q} 25$-ter region was more recently identified as a susceptibility gene for MMD among East Asian population. ${ }^{7-9} \mathrm{We}$ found that a polymorphism in c.14576G > A in RNF213 was identified in $95 \%$ of familial patients with MMD and $79 \%$ of sporadic cases. ${ }^{7}$ Miyatake et al. reported that patients with the polymorphism of c.14576G > A in the RNF213 gene had significantly earlier onset and a more severe form of $\mathrm{MMD}$, such as the presentation of cerebral infarction and posterior cerebral artery stenosis. ${ }^{10}$ To investigate the potential role of RNF213 polymorphism, Liu et al. ${ }^{8}$ investigated the effects of RNF213 suppression on zebra-fish vasculature by generating RNF213 knockdown zebra-fish, demonstrating that zebra-fish lacking RNF213 showed severely abnormal sprouting vessels in the head region, especially from the optic vessels. It was suggested that RNF213 is involved in a novel signaling pathway in intracranial angiogenesis. ${ }^{8}$

\section{Generation of RNF213-deficient mice (RNF213-`)}

Using Cre-lox system, we generated RNF213-deficient mice with C57BL/ 6 background by deleting exon 32 of RNF213, which is the largest exon in the RNF213 gene (Figure 1). ${ }^{12} \mathrm{Fi}-$ nally, heterozygous male and female mice were bred to produce homozygous offspring (homozygous knockout mice, RNF213 ${ }^{-/}$). Genotyping was performed by PCR using specific primers to exon 32 (forward; 5'-CTCAGTGGTGGTGTTGGATG-3', reverse; 5'-CTCTTTCTCGTTGGGACTGC-3') and the deleted allele (forward; 5'-ATAACCTCAGGCACCAATCG-3', reverse; 5'-TCCCTCTAGGCAGGAAGGAT-3'). ${ }^{12}$ The complete removal of RNF213 exon 32 from genomic DNA was confirmed in RNF213\% (Figure 2A). Both homozygous mutant $\left(R N F 213^{-/}\right)$and heterozygous mutant mice $\left(R N F 213^{-/+}\right)$were born and grew normally.

\section{Temporal changes of vascular anatomy in RNF213\%-}

Then we performed magnetic resonance angiography (MRA) using a dedicated small animal scanner with a 50-mm bore operating at 9.4 Tesla field strengths (AV400WV, Bruker BioSpin). ${ }^{12}$ A high resolution three-dimensional gradient-echo time-of- 


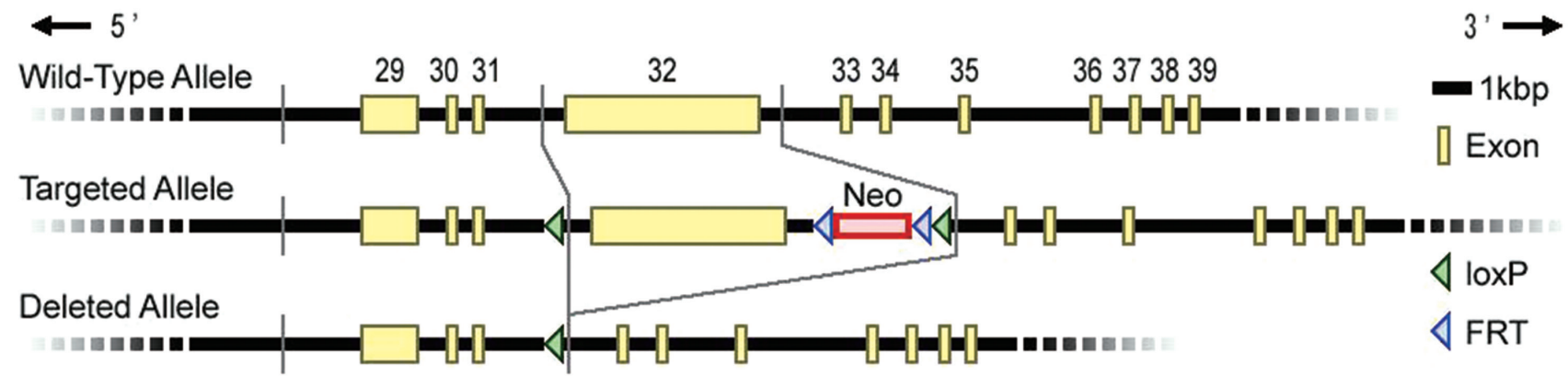

Figure 1. Gene construct of RNF213-deficient mice. Conventional knockout mice were generated by the Cre-lox system.

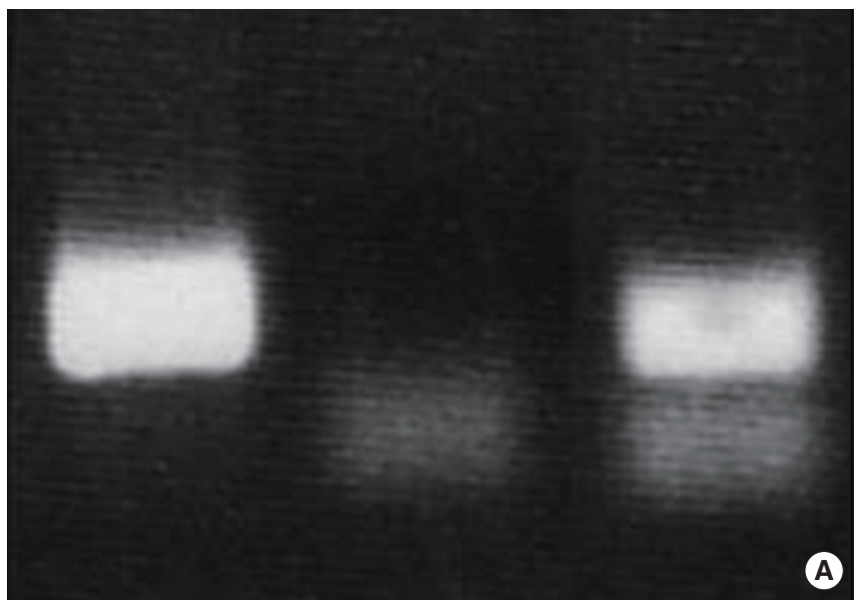

Wt.

RNF213

RNF213-1+

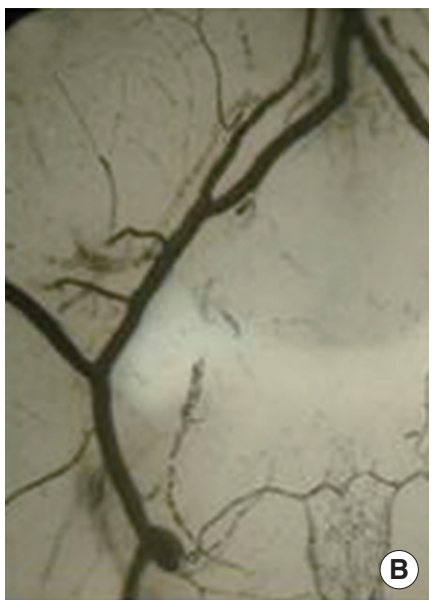

Wt.

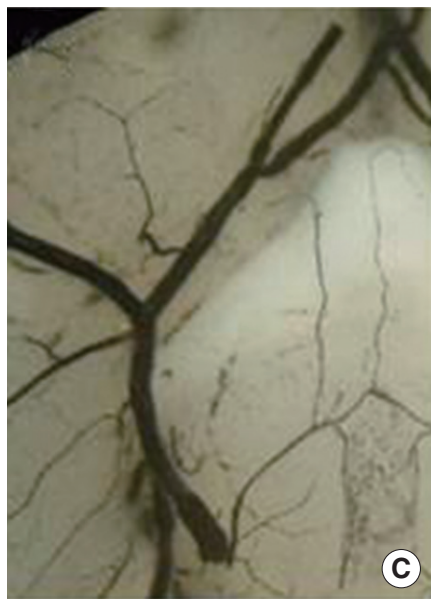

RNF213

Figure 2. (A) Polymerase chain reaction (PCR) genotyping of wild-type mice (Wt.), homozygous RNF213- deficient mice (RNF213/) and heterozygous RNF213- deficient mice $\left(R N F 213^{\prime /}\right)$. (B, C) Microscopic view of the base of the brain at 16 weeks demonstrated no difference in the vascular structure of the circle of Willis between RNF213\% and Wt.

flight MRA sequence was used to acquire MRA images. As a result, no significant difference was observed in the MRA findings of cervical/intracranial arteries between $R N F 213^{-\%}$ and wild-type littermates (Wt.) from 32 to 64 weeks of age. ${ }^{12}$ The anatomy of the circle of Willis was evaluated by a trans-cardiac injection of carbon black dye in RNF213 ${ }^{--}$and Wt. (Figure 2B, C). No significant difference was observed in the structure of the major arteries at the base of the brain between RNF213\% and $\mathrm{Wt}$. at 16 weeks of age (Figure 2B, C). Vessel diameter was sustained at 40 weeks of age in RNF $213^{-/}$, and no steno-occlusive changes were observed around the terminal portion of the internal carotid artery. An abnormal vascular network did not develop at the base of the brain in $R N F 213^{-/ 2} .12$

\section{Modification of vascular remodeling in RNF213\%- after carotid artery ligation}

We also evaluated the histo-pathological characteristics of the vascular wall structure in $R N F 213^{-/}$, and showed no apparent abnormality in mutant mice including intimal hyperplasia or medial layer thinness, both of which are the characteristic find- ings of MMD. Thus we employed common carotid artery (CCA) ligation model, which reproducibly induces arterial wall hyperplasia. ${ }^{17}$ After CCA ligation, Wt. showed temporary hyperplasia of the intima and medial layers (Figure $3 \mathrm{~A}$ ), which was consistent with previous reports; ${ }^{18}$ however, $R N F 213^{-/-}$did not exhibit temporary hyperplasia of the intima and medial layers at the same time point (Figure 3B). Both the intima and medial layers were significantly thinner in $R N F 213^{-/}$than in Wt. 14 days after CCA ligation, while no significant difference was observed in vascular wall thickness 7,21 , and 28 days after CCA ligation. ${ }^{12}$

\section{Role of RNF213 polymorphism in MMD}

Our results demonstrated that $R N F 213^{-/}$grew normally, and no significant difference was observed in MRA findings or the anatomy of the circle of Willis between $R N F 213^{-/}$and Wt. under normal conditions. The histo-pathological characteristics of the vascular wall structure in $R N F 213^{-/-}$showed no apparent abnormality, thus it is conceivable that an abnormality in RNF213 does not sufficiently induce MMD. Recently, Kobayashi and colleagues alternatively generated $R N F 213^{-/}$and suggested that 

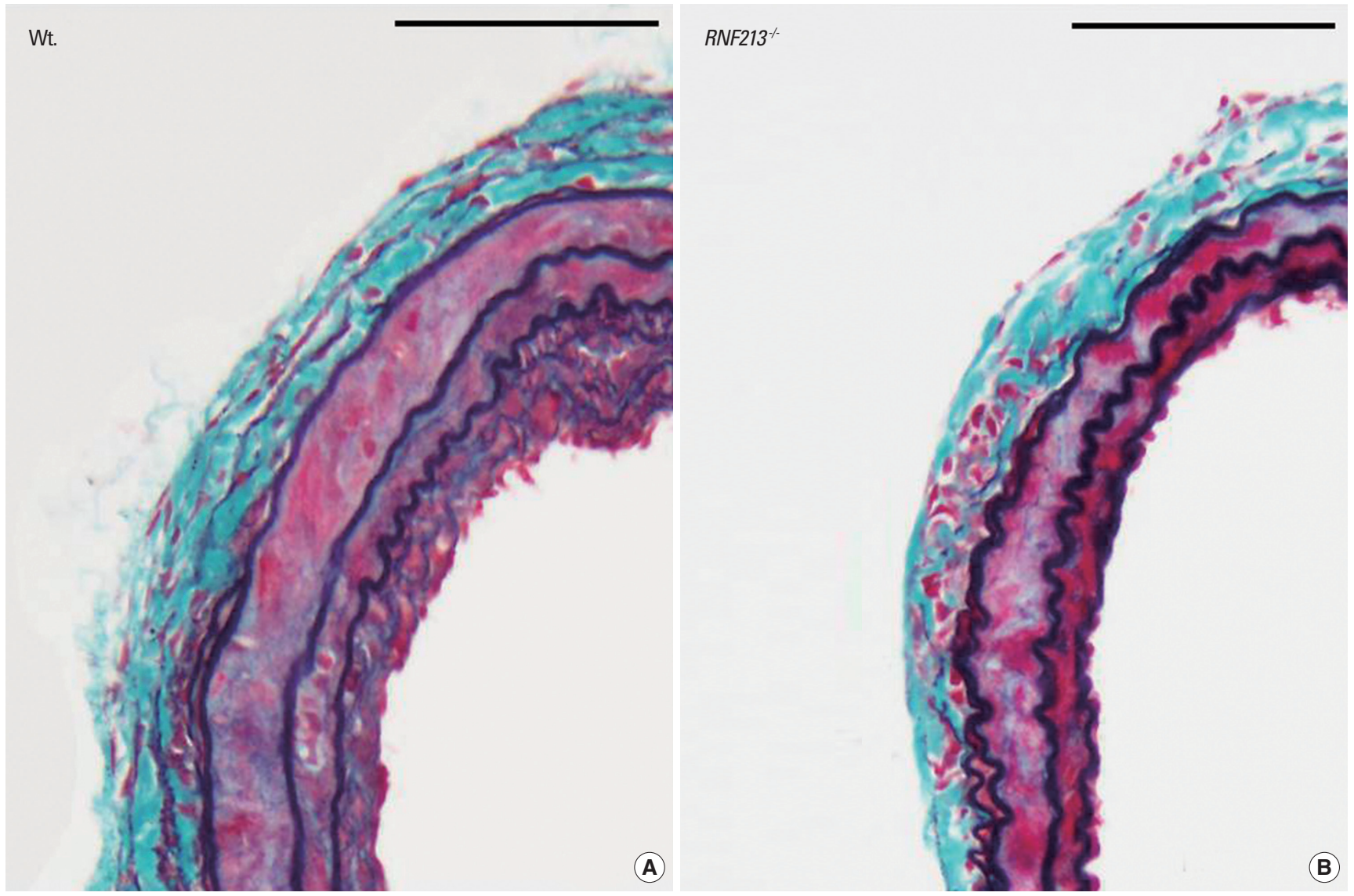

Figure 3. Photo-microscopic view of the wall of the common carotid artery (CCA) 14 days after CCA ligation (High-power, Elastica-Masson staining). Only Wt. exhibited temporary intimal hyperplasia and the medial layer after CCA ligation (Scale bar: $50 \mu \mathrm{m})$.

target disruption of RNF213 improved glucose tolerance by protecting inlet $\beta$ cells when they are mated with Akita diabetic mice, but they also failed to detect any cerebrovascular pathology mimicking MMD, ${ }^{11}$ which is consistent with our result.

Although it is still undetermined whether the polymorphism of c.14576G $>\mathrm{A}$ in patients with MMD is a loss of function mutation or a gain of function mutation, the initial report of knockout zebra-fish by Liu et al. ${ }^{8}$ suggested that it could be a loss of function mutation. In our study, we did not observe any evidence of the characteristic findings of MMD in RNF213 under normal physiological conditions until 64 weeks of age. However, following CCA ligation, which reproducibly induced temporary hyperplasia of the adjacent vascular wall, ${ }^{17,18}$ we showed that the medial layer of CCA was significantly thinner in RNF$213^{-/}$than in Wt. 14 days after CCA ligation, which matched one of the histological characteristic findings of MMD. ${ }^{2}$ Therefore, RNF213 deficiency could lead to vascular fragility including medial thinness, which may make vessels more vulnerable to hemodynamic stress and secondary insults, which facilitates the development of MMD. Furthermore, it is conceivable that secondary insults in addition to RNF213 abnormality, such as an autoimmune response, ${ }^{19,20}$ infection/inflammation, and radiation $^{2}$ may be necessary for the development of MMD. In fact, the incidence rate of MMD was shown to be as low as 0.53 per 100,000 population in Japan, ${ }^{21}$ while $1 \%$ of the Japanese population carries the polymorphism of $\mathrm{c} .14576 \mathrm{G}>\mathrm{A}$ in the RNF213 gene, ${ }^{8}$ again suggesting the importance of additional insult to induce MMD. This observation could be illustrated as 'two-hit theory', as is also true in a variety of disorders (Figure 4).

\section{Possibility of medial thinness as an initial histo-patholohical change in MMD}

Autopsy studies of MMD have shown that the outer diameter, as well as intra-luminal diameter, of the affected arteries of the circle of Willis is smaller in MMD. ${ }^{22}$ Intraoperative finding also suggested smaller outer diameter of the terminal ICA in $M M D .{ }^{23}$ More recently, the arterial constrictive remodeling with early narrowing of outer diameter of the ICA was proposed to be the intrinsic pathogenesis of MMD. Based on their finding of three-dimensional constructive interference in steady-state magnetic resonance imaging demonstrating that marked narrowing of the outer vascular diameter may precede internal vas- 


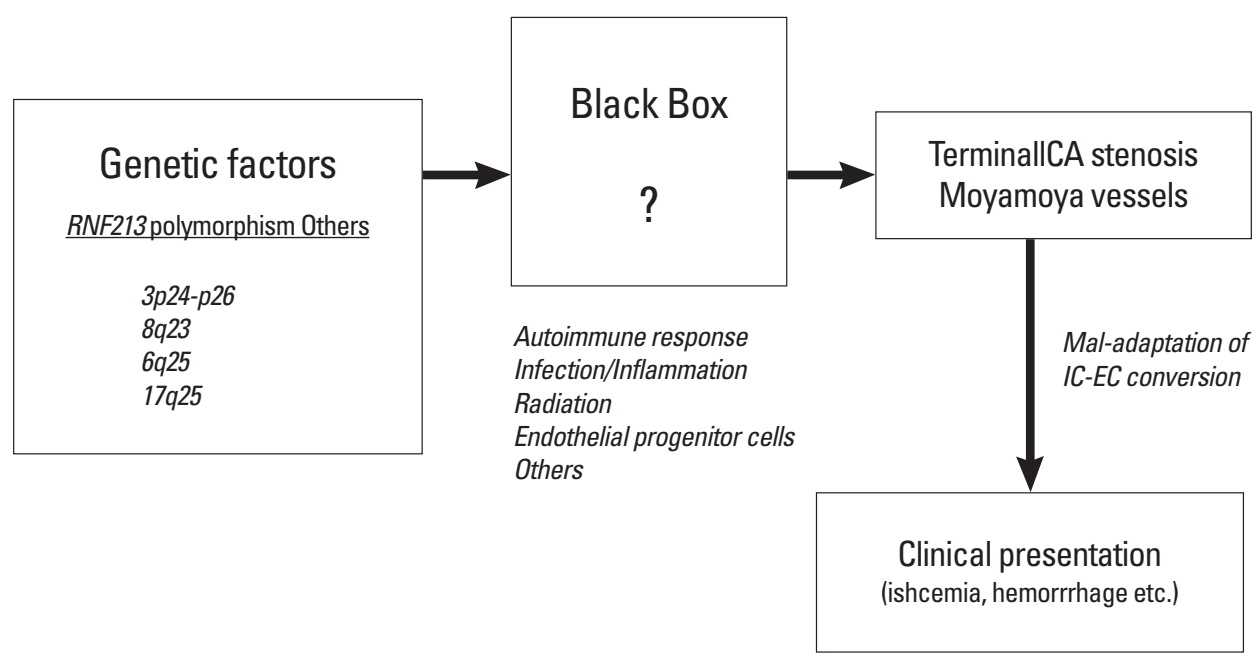

Figure 4. Demographic view of the possible mechanism underlying the development of moyamoya disease. IC-EC conversion: Internal carotid-external carotid conversion as a compensatory physiological reorganization system for moyamoya disease.

cular stenotic progression, ${ }^{24}$ it could be alternatively possible that the thinness of the entire vascular wall in $R N F 213^{-/}$might represent the very early finding of the constrictive remodeling as a characteristic of MMD.

\section{Possibility RNF213 polymorphism as a gain of function mutation}

Alternatively, we should consider another possibility that the RNF213 polymorphism in patients with MMD is a gain of function mutation. ${ }^{25,26}$ Since $R N F 213^{-/}$in our study, which represented a loss of function mutation, did not spontaneously develop MMD under normal conditions, it is also conceivable that the polymorphism of c.14576G > A in RNF213 contributes to the gain of function of the RNF213 product in patients with MMD. ${ }^{12}$ In fact, we obtained conflicting results; the medial layer was significantly thinner in $R N F 213^{-/}$, which is again the characteristic finding of MMD, but temporary intimal hyperplasia was significantly suppressed in $R N F 213^{-/}$during vascular remodeling after CCA ligation, which is not a characteristic of MMD. Further studies using knock-in mice carrying the polymorphism of c. $14576 \mathrm{G}>\mathrm{A}$ in the RNF213 gene would address this important issue.

\section{Biomarkers of MMD: clinical implications}

\section{Expression of angiogenic factors and} pro-inflammatory molecules in MMD

It has been well known that patients with MMD have increased expression of various pro-inflammatory molecules as well as angiogenic factors in serum and/or cerebrospinal fluid (CSF). Increased expression of basic fibroblast growth factor (bFGF) in
CSF from moyamoya patients was considered to be specific and was not thought to be simply related to cerebral ischemia. ${ }^{27}$ The bFGF level was apparently elevated in the patients in whom neovascularization formed from indirect revascularization, suggesting that the bFGF level is a useful indicator to predict the efficacy of indirect revascularization after surgery. ${ }^{27}$ Nanba et al. ${ }^{28}$ alternatively reported the increased expression of hepatocyte growth factor in CSF and intracranial artery in MMD. Regarding serum level of these molecules, Kang and colleagues comprehensively investigated the expression of matrix metalloproteinases, interleukins, and growth factors, and they found that patients with MMD exhibited significantly higher plasma concentrations of matrix metalloproteinase (MMP)-9, monocyte chemoattractant protein-1, interleukin- $1 \beta$, vascular endothelial growth factor (VEGF), and platelet-derived growth factor-BB. ${ }^{29}$ In light of the recent report by Park and colleagues demonstrating that moyamoya patients with VEGF polymorphism of the CC genotype of VEGF-634 had better collateral vessel formation after revascularization surgery, increased serum level of VEGF in patients with MMD should participate in the induction of pial synangiosis after indirect revascularization surgery as well as to the development of abnormal vascular networks at the base of the brain. ${ }^{30}$ Alternatively, increased expression of serum MMP-9 may contribute to the spontaneous intracranial hemorrhage in MMD, as well as to the higher risk for cerebral hyperperfusion syndrome after direct revascularization surgery in moyamoya patients. ${ }^{31,32}$

Pharmacological approach to the biomarkers of MMD: clinical application

EC-IC bypass is an established management of $\mathrm{MMD},{ }^{2}$ but 

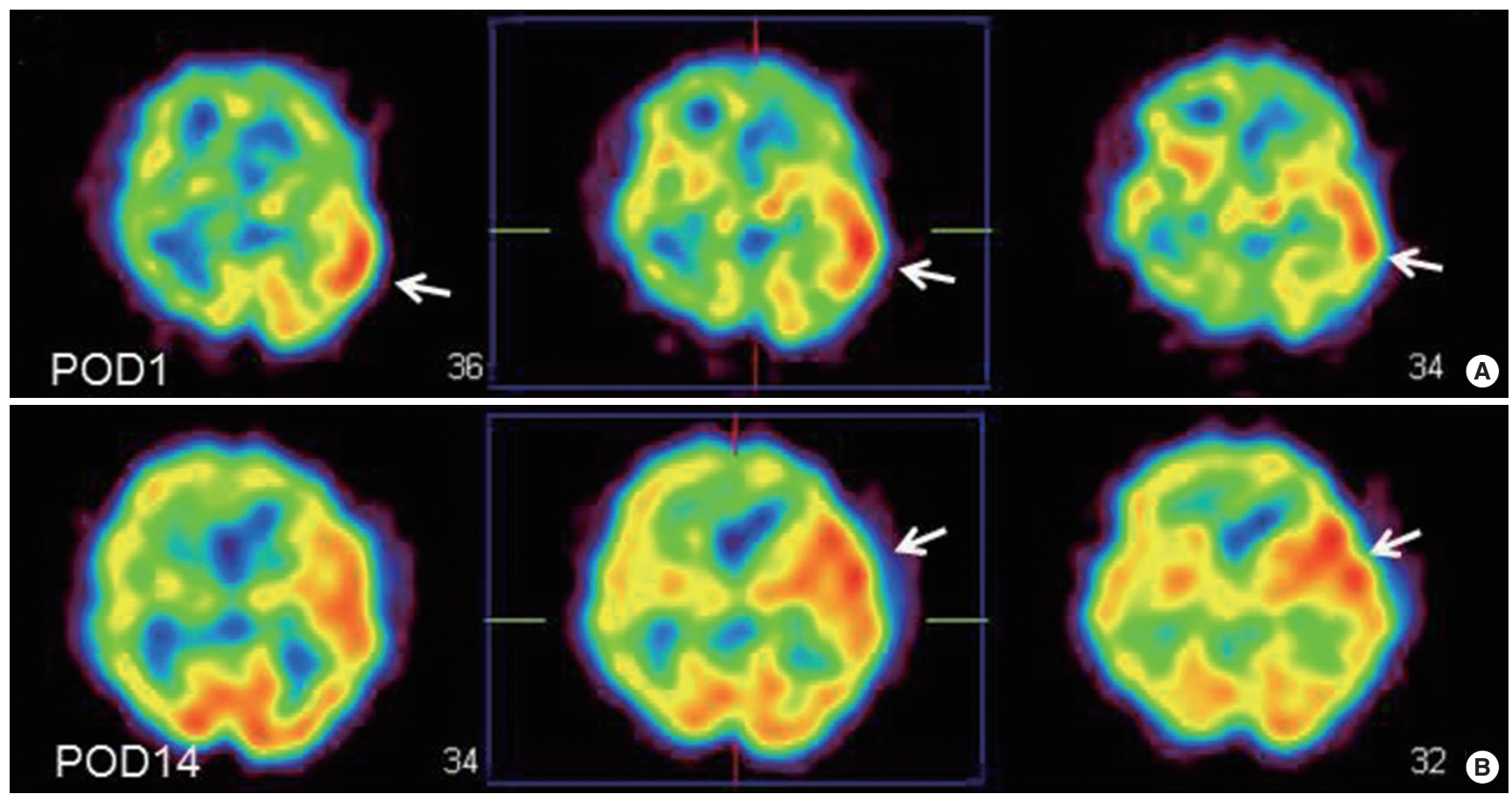

Figure 5. A 43-year old man with ischemic-onset moyamoya disease undergoing left superficial temporal artery-middle cerebral artery anastomosis. Postoperative single-photon emission computed tomography one day $(A)$ and 14 days $(B)$ after surgery demonstrating focal intense increase in cerebral blood flow at the site of the anastomosis (arrows).

cerebral hyperperfusion syndrome (CHS) is one of the critical complications of EC-IC bypass for MMD, which could result in focal neurological deterioration and/or delayed intracranial hemorrhage. ${ }^{32-36}$ Although the mechanism underlying the higher incidence of CHS among patients with MMD has been undetermined, intrinsic vulnerability of vascular structure including blood-brain barrier was considered to lead to vasogenic edema and hemorrhagic conversion following revascularization to the chronic ischemic brain. ${ }^{31}$ Since MMP-9 is known to participate in edema formation and hemorrhagic conversion after cerebral ischemia-reperfusion injury, ${ }^{37,38}$ it was conceivable that the increased expression of MMP-9 may contribute in part to CHS in moyamoya patients. Minocycline hydrochloride was known to play a role in blocking MMP-9, ${ }^{39}$ and is also known to have a neuroprotective effect against ischemic brain injury, ${ }^{40}$ thus we attempted to use minocycline perioperatively to prevent $\mathrm{CHS}{ }^{41}$ The result showed that minocycline significantly reduced the incidence of focal neurological deterioration due to CHS after EC-IC bypass for $\mathrm{MMD}^{41}$ indicating that increased MMP-9 expression in MMD could participate in the pathology of postoperative CHS. This representative 43-year old man with ischemic-onset MMD underwent left EC-IC bypass, and demonstrated significant focal increase in cerebral blood flow at the site of the anastomosis which prolonged over 2 weeks (Figure 5). He was managed by intravenous administration of mi-
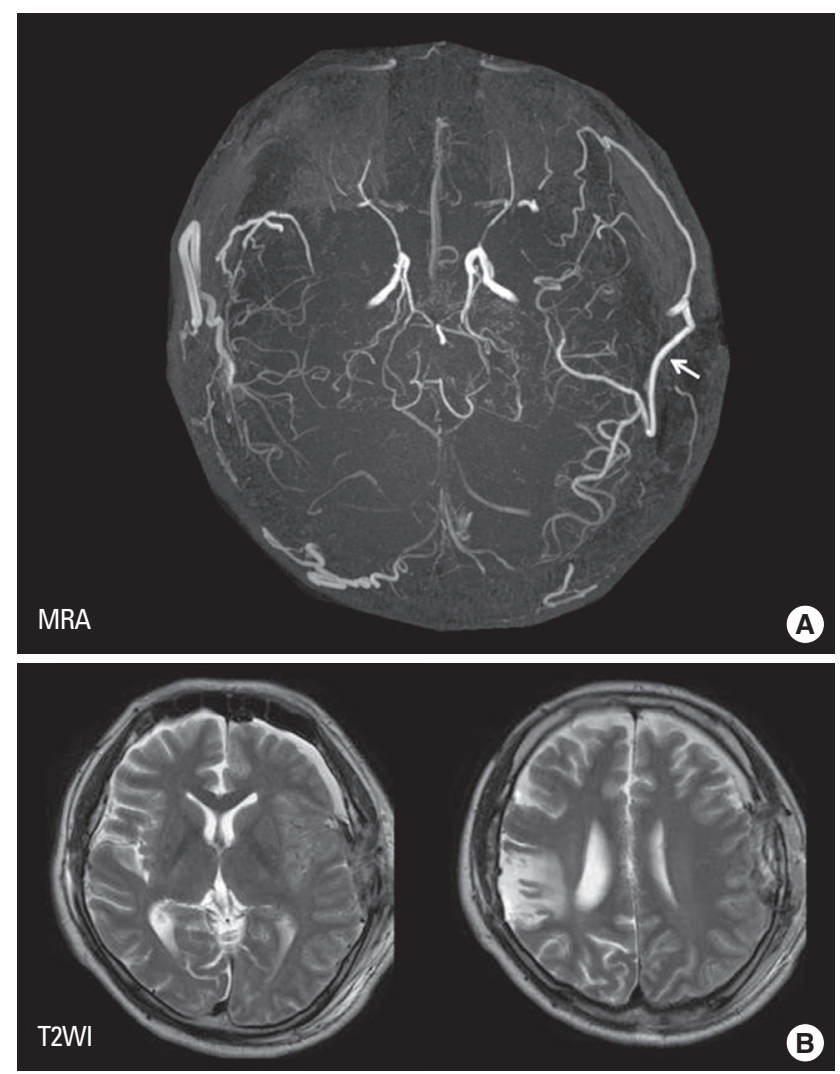

Figure 6. Postoperative magnetic resonance (MR) angiography (A) and MR imaging of T2-wighted images (B) indicating patent bypass (arrow in A) and no evidence of vasogenic edema $(B)$ 
nocycline hydrochloride ( $200 \mathrm{mg} /$ day) under strict blood pressure control, and did not suffer any neurological deterioration during the perioperative period. Postoperative magnetic resonance imaging showed no evidence of vasogenic edema (Figure 6), and he was discharged without neurological deficit. We consider that minocycline has potential role for preventing cerebral hyperperfusion to be symptomatic, by blocking MMP-9 in the acute stage after EC-IC bypass. Future investigation of various biomarkers related to MMD may further improve the outcome of MMD.

\section{Conclusions}

Although the etiology of MMD remains unknown, recent advances have been made in understanding the molecular biology and pathophysiology of this rare entity. Genome-wide and locus-specific association studies identified RNF213 as an important susceptibility gene of MMD. Ongoing research using genetically engineered mice lacking RNF213 by homologous recombination may provide new prospects for the basic research of this rare entity. Alternatively, variety of biomarkers are known to be involved in MMD, and an increased expression of angiogenic factors and pro-inflammatory molecules such as vascular endothelial growth factors and MMP-9 could be the therapeutic target in the future.

\section{Refereces}

1. Suzuki J, Takaku A. Cerebrovascular "moyamoya” disease. Disease showing abnormal net-like vessels in base of brain. Arch Neurol 1969;20:288-299.

2. Research Committee on the Pathology and Treatment of Spontaneous Occlusion of the Circle of Willis; Health Labour Sciences Research Grant for Research on Measures for Infractable Diseases. Guidelines for diagnosis and treatment of moyamoya disease (Spontaneous Occlusion of the Circle of Willis). Neurol Med Chir (Tokyo) 2012;52:245-266.

3. Ikeda H, Sasaki T, Yoshimoto T, Fukui M, Arinami T. Mapping of a familial moyamoya disease gene to chromosome 3p24.2p26. Am J Hum Genet 1999;64:533-537.

4. Inoue TK, Ikezaki K, Sasazuki T, Matsushima T, Fukui M. Linkage analysis of moyamoya disease on chromosome 6.J Child Neurol 2000;15:179-182.

5. Sakurai K, Horiuchi Y, Ikeda H, Ikezaki K, Yoshimoto T, Fukui $\mathrm{M}$, et al. A novel susceptibility locus for moyamoya disease on chromosome 8q23.J Hum Genet 2004;49:278-281.

6. Yamauchi T, Tada M, Houkin K, Tanaka T, Nakamura Y, Kuroda S, et al. Linkage of familial moyamoya disease (spontaneous occlusion of the circle of Willis) to chromosome 17q25. Stroke 2000;31:930-935.

7. Kamada F, Aoki Y, Narisawa A, Abe Y, Komatsuzaki S, Kikuchi A, et al. A genome-wide association study identifies RNF213 as the first Moyamoya disease gene. J Hum Genet 2011;56:3440.

8. Liu W, Morito D, Takashima S, Mineharu Y, Kobayashi H, Hitomi $\mathrm{T}$, et al. Identification of RNF213 as a susceptibility gene for moyamoya disease and its possible role in vascular development. PLoS One 2011;6:e22542.

9. Wu Z, Jiang H, Zhang L, Xu X, Zhang X, Kang Z, et al. Molecular analysis of RNF213 gene for moyamoya disease in the Chinese Han population. PLoS One 2012;7:e48179.

10. Miyatake S, Miyake N, Touho H, Nishimura-Tadaki A, Kondo Y, Okada I, et al. Homozygous c.14576G > A variant of RNF213 predicts early-onset and severe form of moyamoya disease. Neurology 2012;78:803-810.

11. Kobayashi H, Yamazaki S, Takashima S, Liu W, Okuda H, Yan $\mathrm{J}$, et al. Ablation of Rnf213 retards progression of diabetes in the Akita mouse. Biochem Biophys Res Commun 2013;432: 519-525.

12. Sonobe S, Fujimura M, Niizuma K, Nishijima Y, Ito A, Shimizu $\mathrm{H}$, et al. Temporal profile of the vascular anatomy evaluated by 9.4-tesla magnetic resonance angiography and histopathological analysis in mice lacking RNF213; a susceptibility gene for moyamoya disease. Brain Res 2014;1552:64-71.

13. Oka K, Yamashita M, Sadoshima S, Tanaka K. Cerebral haemorrhage in Moyamoya disease at autopsy. Virchows Arch. A Pathol Anat Histol 1981;392:247-261.

14. Takagi Y, Kikuta K, Nozaki K, Hashimoto N. Histological features of middle cerebral arteries from patients treated for Moyamoya disease. Neurol Med Chir (Tokyo) 2007;47:1-4.

15. Yamashita M, Oka K, Tnaka K. Histopathology of the brain vascular networks in moyamoya disease. Stroke 1983;24:19601967.

16. Fujimura M, Tominaga T. Lessons learned from moyamoya disease: outcome of direct/Indirect revascularization surgery for 150 affected hemispheres. Neurol Med Chir (Tokyo) 2012; 52:327-332.

17. Kumar A, Lindner V. Remodeling with neointima formation in the mouse carotid artery after cessation of blood flow. Arterioscler Thromb Vasc Biol 1997; 17:2238-2244.

18. Godin D, Ivan E, Johnson C, Magid R, Galis ZS, Remodeling of carotid artery is associated with increased expression of matrix metalloproteinases in mouse blood flow cessation model. Circulation 2000;102:2861-2866.

19. Kim SJ, Heo KG, Shin HY, Bang OY, Kim GM, Chung CS, et al. Association of thyroid autoantibodies with moyamoya-type 
cerebrovascular disease: a prospective study. Stroke 2010;41: 173-176.

20. Li H, Zhang ZS, Dong ZN, Ma MJ, Yang WZ, Han C, et al. Increased thyroid function and elevated thyroid autoantibodies in pediatric patients with moyamoya disease: a case-control study. Stroke 2011;42:1138-1139.

21. Kuriyama S, Kusaka Y, Fujimura M, Wakai K, Tamakoshi A, Hashimoto S, et al. Prevalence and clinicoepidemiological features of moyamoya disease in Japan: findings from a nationwide epidemiological survey. Stroke 2008;39:42-47.

22. Fukui M, Kono S, Sueishi K, Ikezaki K. Moyamoya disease. Neuropathology 2000; 20(Suppl):S61-S64.

23. Kuroda S, Houkin K. Moyamoya disease: current concepts and future perspectives. Lancet Neurol 2008; 7:1056-1066.

24. Kaku Y, Morioka M, Ohmori Y, Kawano T, Kai Y, Fukuoka H, et al. Outer-diameter narrowing of the internal carotid and middle cerebral arteries in moyamoya disease detected on 3D constructive interference in steady-state MR image: is arterial constrictive remodeling a major pathogenesis? Acta Neurochir (Wien) 2012;154:2151-2157.

25. Hitomi T, Habu T, Kobayashi H, Okuda H, Harada KH, Osafune $\mathrm{K}$, et al. Downregulation of Securin by the variant RNF213 R4810K (rs112735431, G>A) reduces angiogenic activity of induced pluripotent stem cell-derived vascular endothelial cells from moyamoya patients. Biochem Biophys Res Commun 2013a;438:13-19.

26. Hitomi T, Habu T, Kobayashi H, Okuda H, Harada KH, Osafune $\mathrm{K}$, et al. The moyamoya disease susceptibility variant RNF213 R4810K (rs112735431) induces genomic instability by mitotic abnormality. Biochem Biophys Res Commun 2013b; 439:419-426.

27. Yoshimoto T, Houkin K, Takahashi A, Abe H. Angiogenic factors in moyamoya disease. Stroke 1996;27:2160-2165.

28. Nanba R, Kuroda S, Ishikawa T, Houkin K, Iwasaki Y. Increased expression of hepatocyte growth factor in cerebrospinal fluid and intracranial artery in moyamoya disease. Stroke 2004;35: 2837-2842.

29. Kang HS, Kim JH, Phi JH, Kim YY, Kim JE, Wang KC, et al. Plasma matrix metalloproteinases, cytokines and angiogenic factors in moyamoya disease. J Neurol Neurosurg Psychiatry 2010;81:673-678.

30. Park YS, Jeon YJ, Kim HS, Chae KY, Oh SH, Han IB, et al. The role of VEGF and KDR polymorphisms in moyamoya disease and collateral revascularization. PLoS One 2012;7:e47158.

31. Fujimura M, Watanabe M, Narisawa A, Shimizu H, Tominaga T. Increased expression of serum Matrix Metalloproteinase- 9 in patients with moyamoya disease. Surg Neurol 2009;72:476480.
32. Fujimura M, Shimizu H, Inoue T, Mugikura S, Saito A, Tominaga T. Significance of focal cerebral hyperperfusion as a cause of transient neurologic deterioration after EC-IC bypass for moyamoya disease: comparative study with non-moyamoya patients using 123I-IMP-SPECT. Neurosurgery 2011;68:957965.

33. Fujimura M, Kaneta T, Mugikura S, Shimizu H, Tominaga T. Temporary neurologic deterioration due to cerebral hyperperfusion after superficial temporal artery-middle cerebral artery anastomosis in patients with adult-onset moyamoya disease. Surg Neurol 2007;67:273-282.

34. Kim JE, Oh CW, Kwon OK, Park SQ, Kim SE, Kim YK. Transient hyperperfusion after superficial temporal artery/middle cerebral artery bypass surgery as a possible cause of postoperative transient neurological deterioration. Cerebrovasc Dis 2008; 25:580-586

35. Fujimura M, Mugikura S, Kaneta T, Shimizu H, Tominaga T. Incidence and risk factors for symptomatic cerebral hyperperfusion following superficial temporal artery-middle cerebral artery anastomosis in patients with moyamoya disease. Surg Neurol 2009;71:442-447.

36. Uchino H, Kuroda S, Hirata K, Shiga T, Houkin K, Tamaki N. Predictors and clinical features of postoperative hyperperfusion after surgical revascularization for moyamoya disease: a serial single photon emission $\mathrm{CT} /$ positron emission tomography study. Stroke 2012;43:2610-2616.

37. Fujimura M, Gasche Y, Morita-Fujimura Y, Massengale J, Kawase $\mathrm{M}$, Chan PH. Early appearance of activated matrix metalloproteinase- 9 and blood-brain barrier disruption in mice after focal cerebral ischemia and reperfusion. Brain Res 1999;842:92100.

38. Montaner J, Molina CA, Monasterio J, Abilleira S, Arenillas JF, Ribó M, et al. Matrix metalloproteinase-9 pretreatment level predicts intracranial hemorrhagiccomplications after thrombolysis in human stroke. Circulation 2003;107:598-603.

39. Yong VW, Wells J, Giuliani F, Casha S, Power C, Metz LM. The promise of minocycline in neurology. Lancet Neurol 2004;3: 744-751.

40. Yrjänheikki J, Tikka T, Keinänen R, Goldsteins G, Chan PH, Koistinaho J. A tetracycline derivative, minocycline, reduces inflammation and protects against focal cerebral ischemia with a wide therapeutic window. Proc Natl Acad Sci US A 1999;96: 13496-13500.

41. Fujimura M, Niizuma K, Inoue T, Sato K, Endo H, Shimizu $H$, et al. Minocycline prevents focal neurologic deterioration due to cerebral hyperperfusion after extracranial-intracranial bypass for moyamoya disease. Neurosurgery 2014;74:163-170. 